J. Beerten, G. Bergna Diaz, S. D'Arco, and J.A. Suul, "Comparison of small-signal dynamics in MMC and two-level VSC HVDC transmission schemes," in Proc. 2016 IEEE International Energy Conference (ENERGYCON) 2016, Leuven, Belgium, 4-8 Apr. 2016, 6pp.

Digital Object Identifier: 10.1109/ENERGYCON.2016.7514048

URL:

http://ieeexplore.ieee.org/xpls/abs_all.jsp?arnumber $=7514048$

(C) 2016 IEEE. Personal use of this material is permitted. Permission from IEEE must be obtained for all other users, including reprinting/ republishing this material for advertising or promotional purposes, creating new collective works for resale or redistribution to servers or lists, or reuse of any copyrighted components of this work in other works. 


\section{Comparison of Small-Signal Dynamics in MMC and Two-Level VSC HVDC Transmission Schemes}

\author{
Jef Beerten* \\ *Dept. Electrical Engineering \\ KU Leuven - EnergyVille \\ Heverlee-Leuven, Belgium \\ jef.beerten@esat.kuleuven.be
}

\author{
Gilbert Bergna Diaz ${ }^{\dagger}$ \\ Salvatore D'Arco ${ }^{\dagger}$ \\ ${ }^{\dagger}$ SINTEF Energy Research \\ Trondheim, Norway \\ gilbert.bergna-diaz@sintef.no \\ salvatore.darco@sintef.no
}

\author{
Jon Are Suul ${ }^{\dagger \ddagger}$ \\ ${ }^{\ddagger}$ Dept. Electric Power Engineering \\ NTNU \\ Trondheim, Norway \\ jon.are.suul@ntnu.no
}

\begin{abstract}
Small-signal stability studies of power systems including Voltage Source Converter (VSC) HVDC transmission schemes are commonly based on state-space models representing two-level converters and their related control loops. However, an increasing share of HVDC links are based on Modular Multilevel Converters (MMCs), which present additional dynamics and control loops associated with their internal capacitive energy storage. MMC-based HVDC systems do not depend on a large dcside capacitor as two- or three-level VSCs, leading to differences in the dynamic interactions between the converter terminals and HVDC cables. This paper identifies and analyzes the differences in small-signal dynamics between two-level VSC- and MMCterminals in HVDC transmission schemes by eigenvalue analysis. In particular, the differences in interaction patterns within a point-to-point HVDC system are investigated by participation factor analysis. The validity of the models used to generate the presented results is verified by time-domain simulations.
\end{abstract}

Index Terms-HVDC Transmission, Modular Multilevel Converters, Small-signal stability, Voltage Source Converters.

\section{INTRODUCTION}

With the growing demand for Voltage Source Converter (VSC)-based HVDC transmission schemes, there has been an increasing attention towards analyzing their small-signal dynamics and their impact on power system stability. Such studies are already well-developed for HVDC systems based on two-and three-level VSCs, and the corresponding models can be directly applied to the study of multi-terminal transmission schemes [1]-[4].

Currently, the Modular Multilevel Converter (MMC) proposed by [5] is emerging as a preferred topology for most VSC-based HVDC transmission schemes. Instead of a large dc-side capacitor as in traditional two-level VSCs, the MMC has independent phases with distributed internal capacitors. Thus, compared to two-level VSCs, the MMC exhibits additional internal energy dynamics which must be represented in

The work of Jef Beerten is funded by a postdoctoral research grant from the Research Foundation - Flanders (FWO).

The work of SINTEF Energy Research in this paper was supported by the project "Protection and Fault Handling in Offshore HVDC Grids," (ProOfGrids), financed by the Research Council of Norway's RENERGI program and the industry partners; EDF, National Grid, Siemens, Statkraft, Statnett, Statoil and NVE: http://www.sintef.no/Projectweb/ProOfGrids/

978-1-4673-8463-6/16/\$31.00 (C) 2016 IEEE order to accurately assess the small-signal stability properties of MMC-based HVDC transmission schemes. The dynamics of the interaction between an HVDC converter terminal and the HVDC cable can also be different for the MMC compared to two-level VSCs due to the significantly reduced equivalent capacitance at the de terminals.

The representation of all internal single-phase dynamics of an MMC results in complicated models that are not suitable for linearization and small-signal power system stability studies. Thus, approaches for developing simplified time-invariant dynamic state-space models of MMCs that can be linearized around a steady-state operating point are being proposed [6], [7]. These modeling approaches are comparable to continuoustime small-signal models for two-level VSCs in terms of implied simplifications, level of detail and accuracy. Although small-signal models can be developed to represent the internal variables of the MMC with different levels of detail, the most important aspect for applications in power system studies is an accurate representation of the interface variables of the converter's ac- and dc-side and their dynamics.

Although the models in [6], [7] are developed for the purpose of system stability studies, they have only been analyzed for single HVDC converter terminals so far. For studying the small-signal dynamics of HVDC transmission schemes, it is also of vital importance to ensure accurate representation of dc cables or transmission lines [8], [9]. However, no previous studies have analyzed the small-signal dynamics of complete MMC-based HVDC transmission schemes in comparison to established models of two-level VSC-based HVDC systems, including a detailed representation of the cable dynamics.

In this paper the small-signal dynamics and stability properties of an HVDC transmission scheme with MMCs, modeled according to the approach from [6], are compared to an equivalent scheme with converter stations based on two-level VSCs. The models also take into account the frequency-dependent characteristics of HVDC cables to ensure accurate representation of the overall system dynamics. In particular, the analysis highlights the influence of the reduced equivalent capacitance at the de terminals in MMC-based HVDC system and the impact of the internal MMC energy dynamics on small-signal interaction modes of HVDC transmission schemes. 


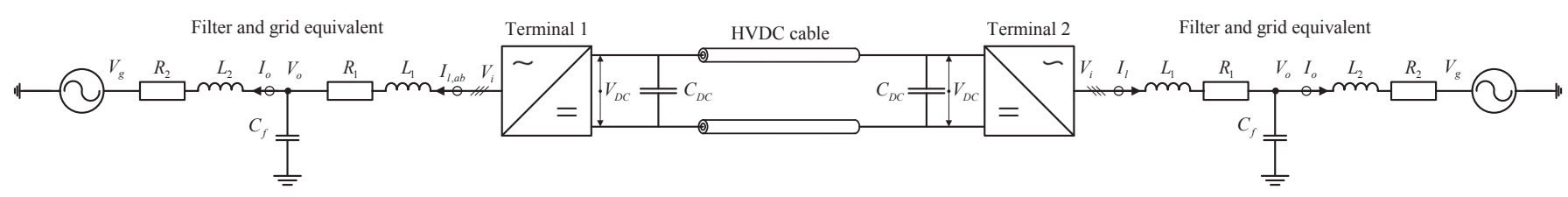

Fig. 1. Overview of investigated system configuration of the point-to-point HVDC transmission scheme.

\section{System CONFIGURATION AND MODELING}

A point-to-point HVDC transmission scheme based on either two-level VSCs or MMC-based converter stations is investigated. The variables and parameters of the HVDC system model are indicated in Fig. 1. As can be inferred from this figure, the same system configuration and the same electrical models of ac- and dc-side elements are used for both configurations in order to facilitate the comparison. Thus, the main difference between the two investigated cases is the topology of the converter itself, its associated control loops and the absence of an explicit large dc-link capacitor in the MMC-based HVDC system.

\section{A. Two-level VSC terminal modeling and control}

The two-level VSC converters are represented by an average model, which can be expressed in per unit values by the power balance equation according to:

$$
v_{i, d} \cdot i_{l, d}+v_{i, q} \cdot i_{l, q}=v_{d c} \cdot i_{d c}
$$

The electrical system on the ac-side of a converter is modeled in a $d q$-Synchronous Reference Frame (SRF). The ac-side interface is formed by an LC filter and a Thevenin equivalent representation of the grid.

The dc-side of the two-level VSC is represented by a capacitor interfaced to the HVDC cable, with the equivalent cable current represented by an equivalent current source $I_{d c, s}$, as shown in Fig. 2. The dc-side of the converter can then be modeled by:

$$
\frac{d}{d t} v_{d c}=\frac{\omega_{b}}{c_{d c}} i_{d c, s}-\frac{\omega_{b}}{c_{d c}} i_{d c}
$$

where $v_{d c}, i_{d c, s}$ and $i_{d c}$ represent respectively the per unit values of the dc-side converter voltage, the dc cable equivalent input current and the current entering the VSC.

The two-level VSC control strategy is illustrated in Fig. 2, and it is based on conventional SRF PI current controllers with decoupling terms. In addition, an outer ac power or dc voltage control loop is used to calculate the reference value for the inner current controller. A compensated modulation strategy has been assumed for the VSC. Hence, the voltage reference $v_{i}^{*}$ resulting from the current control is assumed to be divided by the measured dc voltage $v_{d c}$ to calculate the modulation index $\boldsymbol{m}$. This strategy forces the instantaneous average value of the converter output voltage $v_{i}$ to be approximately equal to $v_{i}^{*}$, provided that PWM delays and switching effects are neglected, as given by:

$$
\boldsymbol{m}=\frac{v_{\boldsymbol{i}}^{*}}{v_{d c}}, \quad v_{i} \approx \boldsymbol{m} \cdot v_{d c} \rightarrow v_{\boldsymbol{i}} \approx v_{\boldsymbol{i}}^{*}
$$

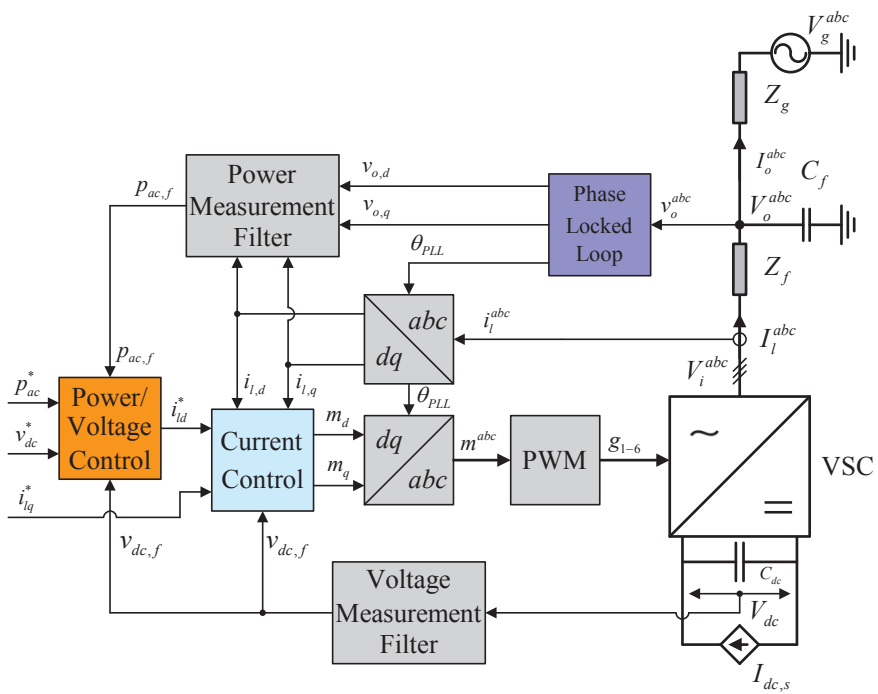

Fig. 2. Configuration and control system for two-level VSC terminal.

Further details on the modeling approach, the applied per unit system and the state-space representation of the control loops including the Phase Locked Loop (PLL) are described in [10].

\section{B. MMC terminal modeling and control}

The MMC terminals have been modeled in accordance with the approach presented in [6], as briefly explained in this section. It should be noted that the ac-side control system from [6] is largely the same as for the two-level VSC and thus, only issues specifically related to the MMC modeling and control are described here.

The MMC models are based on the assumption of a control strategy implemented with compensated modulation strategy, which means that the upper and lower insertion indices $n_{u}$ and $n_{l}$ are calculated as

$$
n_{u}=\frac{-v_{i}^{a b c, *}+v_{c}^{a b c, *}}{v_{u}^{\Sigma}} ; \quad n_{l}=\frac{v_{i}^{a b c, *}+v_{c}^{a b c, *}}{v_{l}^{\Sigma}}
$$

In (4), the output reference voltages $v_{i}^{*}$ and $v_{c}^{*}$, resulting from ac-side current controllers and circulating current controllers respectively, are divided by the measured (or estimated) sum capacitor voltages of the upper and lower arms, $v_{u}^{\Sigma}$ and $v_{l}^{\Sigma}$.

When calculating the insertion indices by (4) instead of using a constant value for the sum arm capacitor voltages (e.g. $\left.V_{d c}^{*}\right)$, the voltages driving the ac-side currents and the circulating currents become approximately equal to their reference 


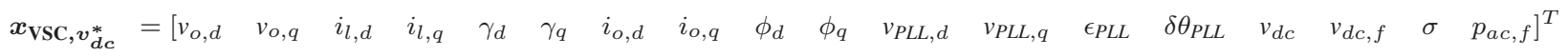

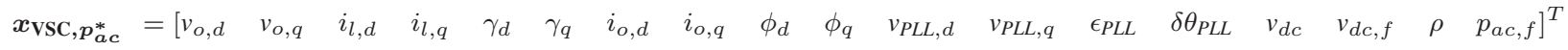

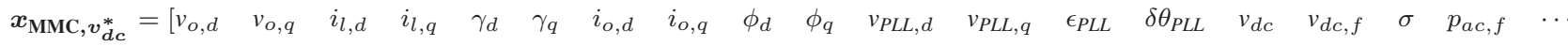

$$
\begin{aligned}
& \left.i_{c, z} \quad w_{\Sigma} \quad \kappa_{\Sigma} \quad \xi_{z}\right]^{T}
\end{aligned}
$$

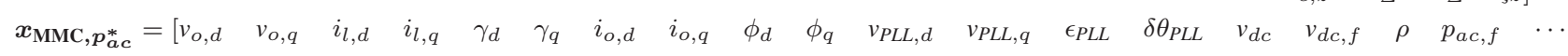

$$
\begin{aligned}
& \left.i_{c, z} \quad w_{\Sigma} \quad \kappa_{\Sigma} \quad \xi_{z}\right]^{T}
\end{aligned}
$$

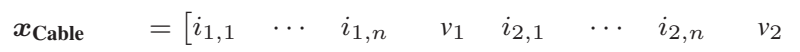

$$
\begin{aligned}
& \left.\begin{array}{llll} 
& & & \\
v_{m-1} & i_{m, 1} & \cdots & i_{m, n}
\end{array}\right]^{T}
\end{aligned}
$$

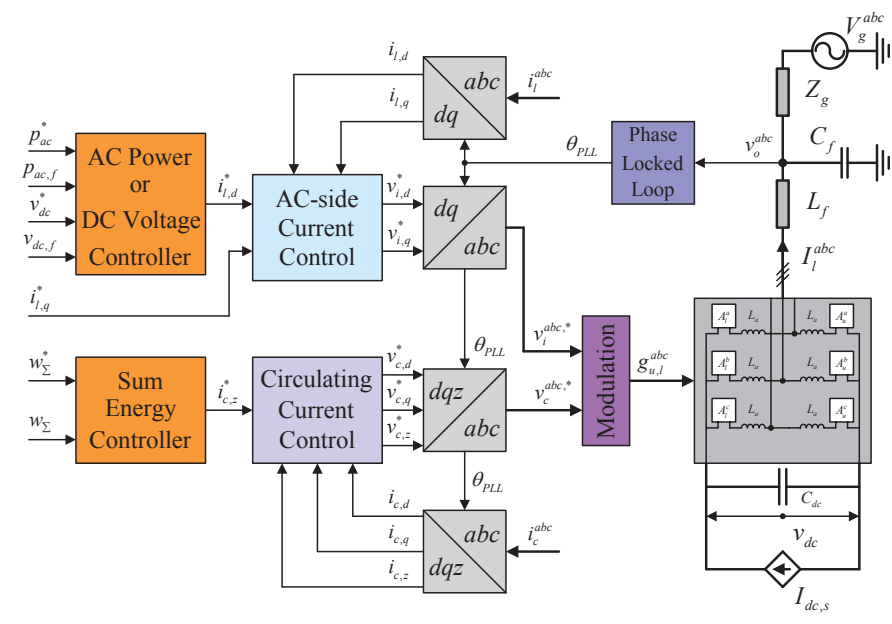

Fig. 3. Overview of configuration and control system for MMC terminal.

values, as indicated in (5)-(6):

$$
\begin{aligned}
& v_{i}^{a b c}=v_{i}^{a b c, *} \rightarrow v_{i, d q} \approx v_{i, d q}^{*} \\
& v_{c}^{a b c}=v_{c}^{a b c, *} \rightarrow v_{c, d q z} \approx v_{c, d q z}^{*} \rightarrow v_{c, z} \approx v_{c, z}^{*}
\end{aligned}
$$

In addition, (4) decouples the zero-sequence dynamics of the energy-sum $w_{\Sigma}$ from the individual energy difference oscillations $w_{\Delta}$. Moreover, it is possible to neglect the effect of the $d q$-components of $v_{c}$ in the energy-sum zero-sequence dynamics, as they are significantly smaller than the zerosequence component $v_{c, z}[6]$.

From these assumptions, a simplified representation of the MMC can be obtained by representing only the zero-sequence dynamics of the energy-sum, $w_{\Sigma}$, and of the circulating current, $i_{c, z}$, which are inherently time-invariant variables in steady state. Thus, the energy difference and the other frequency components of the circulating current can be neglected while still obtaining a model that is accurately representing an MMC-based VSC HVDC station as seen from its ac- and dc-terminals. This model is represented by means of (7)-(8), capturing accurately the macroscopic behavior of the MMC:

$$
\begin{aligned}
& \frac{d w_{\Sigma}}{d t}=\left[-\left(v_{i, d} \cdot i_{l, d}+v_{i, q} \cdot i_{l, q}\right)+4 v_{c, z} \cdot i_{c, z}\right] \frac{\omega_{b}}{8 c_{e q}} \\
& \frac{d i_{c, z}}{d t}=\frac{\omega_{b}}{l_{a}} v_{d c}-\frac{\omega_{b}}{l_{a}} v_{c, z}-\frac{r_{a} \omega_{b}}{l_{a}} i_{c, z}
\end{aligned}
$$

In (7), $c_{e q}$ is the total equivalent capacitance per arm of the MMC (derived from $C_{s m} / \mathrm{N}$ with $C_{s m}$ the submodule capacitance), represented in the ac-side per unit system.

An overview of the control system is depicted in Fig. 3. In addition to the same ac-side control loops as used for the two-level VSCs, the control of the total energy-sum and the zero-sequence circulating currents are explicitly represented in the model. Thus, an outer loop PI controller for the sum energy is providing the reference for an inner loop PI controller for the zero-sequence circulating current. It should be noted that low pass filters are applied on the ac power and dc voltage measurements similarly to the two-level VSC in Fig. 2, although they are not included in the figure for simplicity.

\section{Cable modeling}

The HVDC cables in Fig. 1 are represented according to the frequency-dependent state-space modeling approach proposed in [8]. Thus, a lumped parameter model with multiple parallel RL-branches in addition to multiple pi-sections is used. This model has been shown to accurately represent the internal frequency-dependent dynamics of HVDC cables in the frequency range relevant for small-signal stability analysis. The validity of the model has also been confirmed by direct comparison to a Universal Line Model representation designed for time-domain simulations in [11].

\section{System models}

In order to study the small-signal dynamics for the configuration from Fig. 1, state-space models of the individual converter stations and the HVDC cable, expressed according to (9), have been linearized and assembled to system models according to the same procedure as explained in [8], [9].

$$
\dot{x}_{k}=\mathrm{A} x_{k}+\mathrm{B} u_{k}
$$

The states, $\boldsymbol{x}_{\mathbf{V S C}}$, included in the model for a dc-voltagecontrolled and a power-controlled two-level VSC terminal are listed in (10) and (11) respectively, while the corresponding input vector $\boldsymbol{u}_{\mathbf{V S C}}$ is given by (15). Similarly, the states $\boldsymbol{x}_{\mathbf{M M C}}$ of MMC-based terminals are listed in (12) and (13) respectively, while the input vector for the MMC models is given by (16). In addition to the variables already defined in Figs. 1-3, $\gamma$ represents integrator states of current controllers, $\phi$ represents low pass filter states used for damping of LC-oscillations, all variables with subscripts 'PLL' represent internal states of the PLL as described in [10], $\sigma$ represents the integrator state of dc-voltage controllers and $\rho$ represents the integrator state of ac power controllers. The MMC models have 4 additional 
TABLE I

AC SYSTEM PARAMETERS

\begin{tabular}{|l|cc|}
\hline Rated power & 900 & {$[\mathrm{MW}]$} \\
Ac voltage & 380 & {$[\mathrm{kV}]$} \\
Transformer $u_{k}$ & 0.15 & {$[\mathrm{pu}]$} \\
Ac grid reactance $X_{a c}$ & 17.7 & {$[\Omega]$} \\
Ac grid resistance $R_{a c}$ & 1.77 & {$[\Omega]$} \\
\hline Filter bus capacitance $C_{f}$ & 1.76 & {$[\mu \mathrm{F}]$} \\
\hline
\end{tabular}

TABLE II

CONVERTER PARAMETERS

\begin{tabular}{|l|ccc|}
\hline & 2-level VSC & MMC & \\
\hline Arm inductance $L_{a}$ & - & 84.8 & {$[\mathrm{mH}]$} \\
Arm resistance $R_{a}$ & - & 0.885 & {$[\Omega]$} \\
Phase reactor $L_{1}$ & 83.9 & 41.5 & {$[\mathrm{mH}]$} \\
Phase resistance $R_{1}$ & 0.457 & 0.015 & {$[\Omega]$} \\
Arm capacitance $C_{e q}$ & - & 29.3 & {$[\mu \mathrm{F}]$} \\
Dc capacitance $C_{d c, p t g}$ & 62.7 & 0.63 & {$[\mu \mathrm{F}]$} \\
\hline
\end{tabular}

states compared to the two-level VSC models, where $\kappa_{\Sigma}$ and $\xi_{z}$ are respectively the integrator states of the PI-controllers for the aggregated sum energy $w_{\Sigma}$ and for the equivalent zerosequence circulating current $i_{c, z}$. The per unit grid frequency $\omega_{g}$, which is tracked by the PLLs, is also an input to the models together with the grid voltage amplitude $\left|v_{g}\right|$ and the reference signals for the controllers.

$$
\begin{aligned}
& \boldsymbol{u}_{\mathbf{V S C}}=\left[\begin{array}{llllll}
i_{l, q}^{*} & \left|v_{g}\right| & \omega_{g} & i_{d c, s} & p_{a c}^{*} & v_{d c}^{*}
\end{array}\right]^{T} \\
& \boldsymbol{u}_{\mathbf{M M C}}=\left[\begin{array}{lllllll}
i_{l, q}^{*} & \left|v_{g}\right| & \omega_{g} & i_{d c, s} & p_{a c}^{*} & v_{d c}^{*} & w_{\Sigma}^{*}
\end{array}\right]^{T}
\end{aligned}
$$

The cable is represented by the states $x_{\text {Cable }}$ as given in (14) for a model with $n$ parallel RL-branches per each of the $m$ cascaded pi-sections. The input to the cable model is the voltage at the cable ends as given by (17), which are included as states in the models of the converter stations. The equivalent capacitance of the pi-sections at the ends of the cable is added to the dc-side capacitance of the converter terminals.

$$
\boldsymbol{u}_{\text {Cable }}=\left[\begin{array}{ll}
v_{d c 1} & v_{d c 2}
\end{array}\right]^{T}
$$

When assembling the overall system model, the voltage inputs to the cable model will be states of the converter models while the dc-side currents in the converter models will result from the sum of internal states in the cable model. Thus, these inputs will be eliminated from the system model.

\section{MOdEL VERIFICATION}

The analysis in this paper is based on a generic pointto-point HVDC system from Fig. 1 with either two-level VSCs or MMCs in the converter stations. In this section, the validity of the applied small-signal state-space models used for eigenvalue and participation factor analysis is confirmed by time domain simulations.

\section{A. System parameters}

The investigated configuration is based on a symmetrical monopolar system with a voltage rating of $\pm 320 \mathrm{kV}$ and

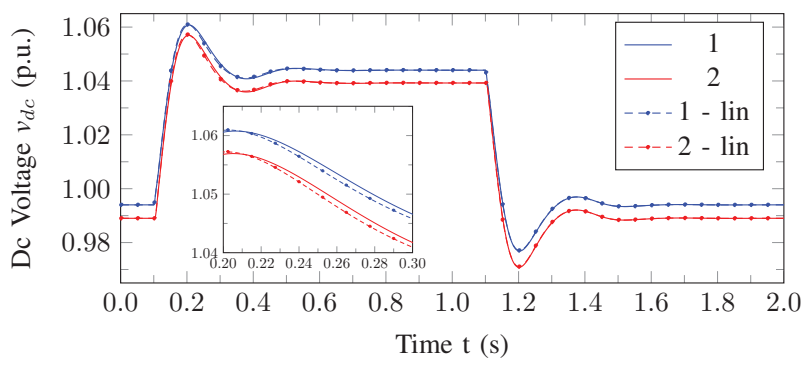

Fig. 4. Two-level VSC time-domain comparison - system response to voltage setpoint change.

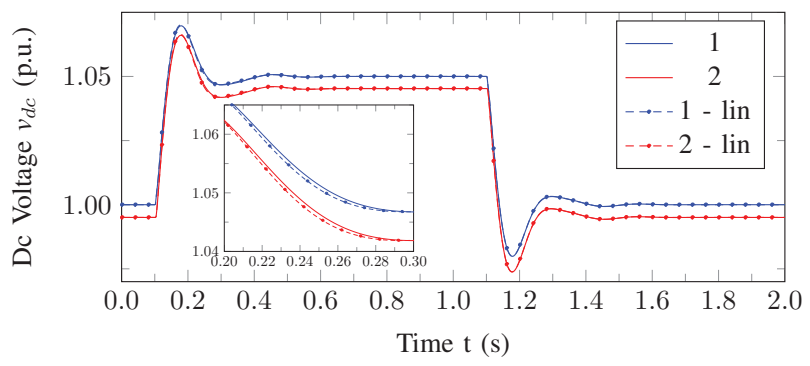

Fig. 5. MMC time-domain comparison - system response to voltage setpoint change.

a power rating of $900 M W$, and uses the data from the HVDC grid test system in [12] for the MMC topology and cable geometry. The ac system data, shown in Tab. I, has been chosen equally for both converters in order to facilitate the comparison of the two different topologies. Likewise, the phase reactor $L_{1}$ in the MMC scheme (Tab. II) have been selected such that the corresponding overall equivalent ac inductance (including $L_{a} / 2$ ) is the same for the two-level VSC and the MMC scheme. A similar remark holds for corresponding resistive part $R_{1}$. The dc-side capacitance in the MMC model (pole-to-ground value $C_{d c, p t g}$ in Tab. II), is merely present to turn the dc-side voltage into a state-variable and has therefore been chosen small, i.e. equal to $1 \%$ of the value in the two-level implementation.

\section{B. Time-domain verification}

The linearised small-signal models have been tested in the time-domain against non-linear three-phase averaged models in MATLAB/Simulink SimPowerSystems. The average models of the MMCs are based on the reduced order nonlinear model derived and linearized in [6], which has been shown to accurately represent the terminal dynamics corresponding to the average modeling approach presented in [13], with each arm of the converter represented as a controlled voltage source.

Fig. 4 shows the step-response of the two-level VSC HVDC point-to-point system to a change in the dc voltage reference. Fig. 5 shows the system response of the MMC-based equivalent. As is clear from the figures, the linearized state-space models are accurately representing the small-signal dynamics of the two investigated systems in a reasonable operating range around the linearization point. Similar accuracy is also 


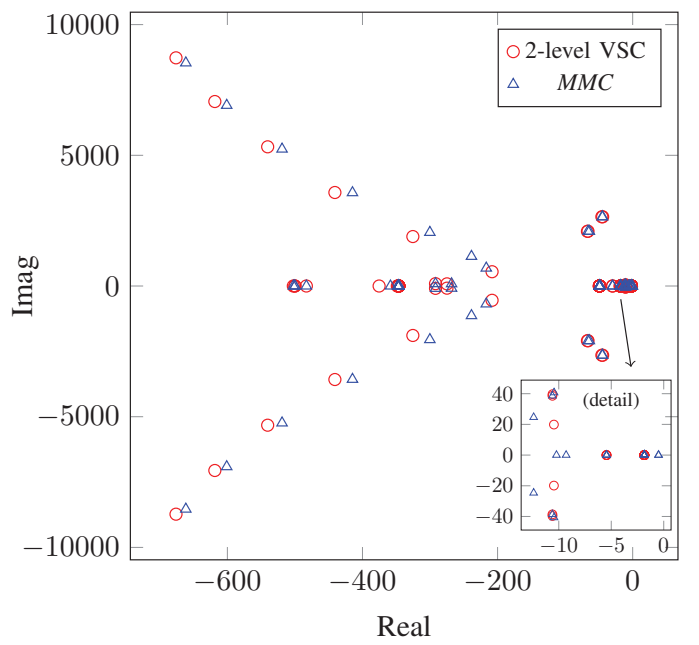

Fig. 6. Eigenvalues of the two-level VSC and MMC-based scheme

obtained for the other state variables in the system models. More details on the time-domain response of the linearized model of the two-level VSC are found in [9].

\section{COMPARISON AND ANALYSIS OF EIGENVALUES AND INTERACTION MODES}

\section{A. Analysis of system eigenvalues}

Fig. 6 shows all the eigenvalues of the two investigated system configurations with real parts over -1000 . The modes that are left out of the picture are either fast real poles and complex conjugate poles associated with well-damped highfrequency cable dynamics. Due to the choice of the ac-side parameters, the poles related to the $\mathrm{LC}$ filter resonances at the ac-side within the frequency range of 300 to $450 \mathrm{~Hz}$ are in similar positions. The MMC shows a number of slow modes, partly introduced by the converter's internal dynamics (e.g. circulating current through the arm inductances). The modes associated to resonances at the dc-side (diagonals with complex conjugate modes with higher damping the higher the frequency) appear at different frequencies.

\section{B. Analysis of interaction modes}

This paper uses the concept of participation factors to identify the modes representing interactions in the system by applying the procedure presented in [8]. These interaction modes are defined as those system modes in which the two converters participate. Using participation factors as defined in [14], let $p_{k i}$ denote the participation factor of state variable $x_{k}$ in mode $i, \boldsymbol{p}_{\boldsymbol{i}} \in \mathbb{R}^{n_{t}}$ the vector with the participation factors for all system states associated with mode $i$, and $\boldsymbol{p}_{\alpha, i} \in \mathbb{R}^{n_{\alpha}}$ the vector for all states of subsystem $\alpha$. A parameter $\eta_{\alpha i}$ is now defined as a measure for the overall participation for each subsystem $\alpha$ in mode $i$ such that

$$
\eta_{\alpha i}=\frac{\left\|p_{\alpha, i}\right\|}{\left\|p_{i}\right\|}
$$

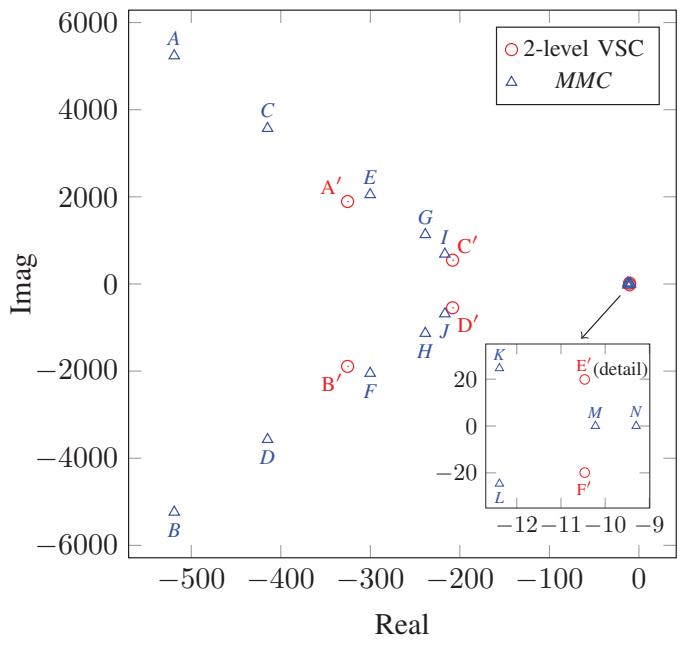

Fig. 7. Interaction modes in two-level VSC vs. MMC-based scheme

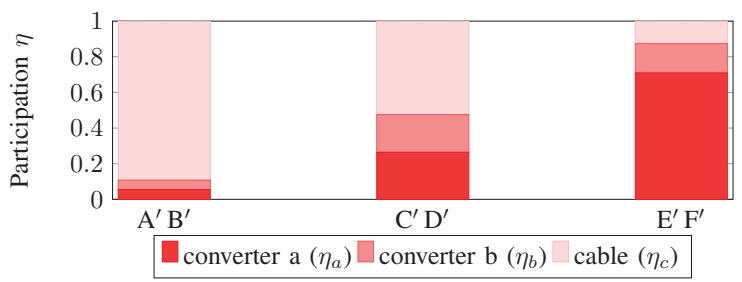

(a)

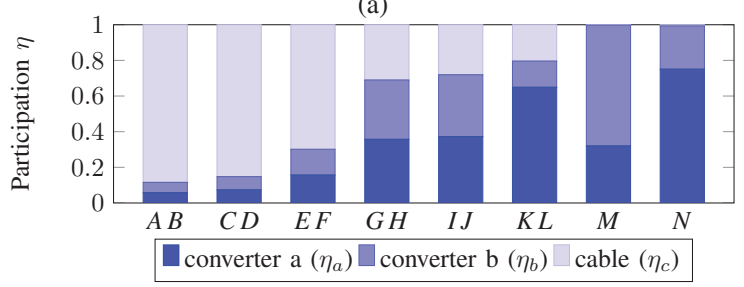

(b)

Fig. 8. Component participation in interacting modes for (a) two-level VSC terminals and (b) MMC terminals.

with $\|\cdot\|$ denoting the $L_{1}$-norm. $\eta_{a i}, \eta_{b i}$ and $\eta_{c i}$ are a measure for the degree to which the two converters and the cable participate in each mode. Using a threshold $\chi$, an interaction mode $i$ is defined as a mode for which both $\eta_{a i}>\chi$ and $\eta_{b i}>\chi$, resulting in a subset of interaction modes $\mathcal{S}$.

Fig. 7 shows the modes resulting from this interaction analysis with a treshold of $5.2 \%$. Fig. 8 shows the overall component participation $\eta$ according to the definition from (18). The MMC-based scheme shows more interaction modes than the two-level VSC-based scheme. The complex conjugate modes $\mathrm{E}^{\prime}, \mathrm{F}^{\prime}$ for the 2-level topology and $K, L$ for the MMC (see Fig. 8 and the subplot in Fig. 7) are interaction modes associated with the power flow in the system and largely linked to $\rho$, the integrator state variable of the dc voltage control. The complex conjugate modes $A$ to $F$ for the $\mathrm{MMC}$ and $\mathrm{A}^{\prime}$ to $\mathrm{D}^{\prime}$ for the two-level VSC, are related to dc resonances with the cable and are to a certain extent linked to the dc voltages at both cable ends. Modes $G$ to $J$ are dc-side interactions that are to a large extent linked with the states associated 


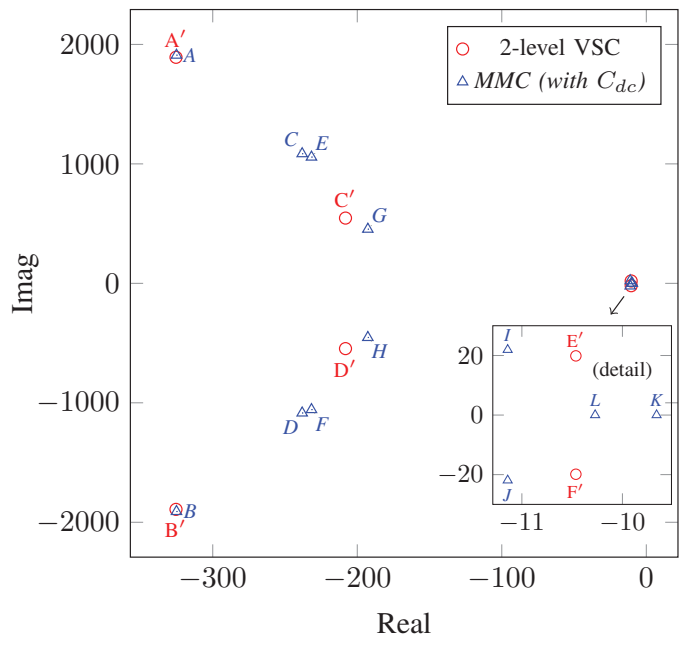

Fig. 9. Interaction modes in two-level VSC vs. MMC-based scheme (with $\left.C_{d c, p t g}=62.7 \mu \mathrm{F}\right)$

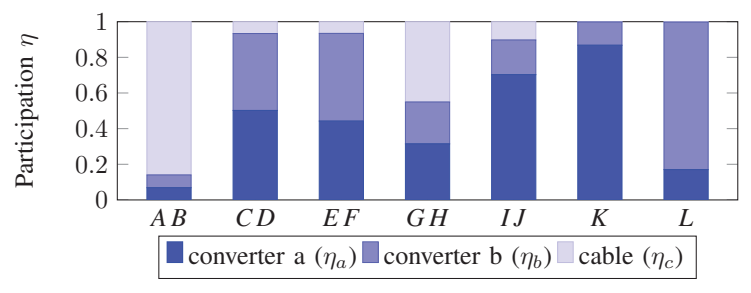

Fig. 10. Component participation in interacting modes for MMC terminals (with $C_{d c, p t g}=62.7 \mu \mathrm{F}$ ).

with the MMC's energy-sum $w_{\Sigma}$ dynamics and the circulating currents $i_{c, z}$. With the same parameters and tuning rules for both converters, the dynamics are similar for both sides of the link. The real poles $M, N$ are linked to $\xi_{z}$, the integrator states of the PI-controllers for the zero-sequence circulating current, and indicate current dynamics that are the same in two MMCs.

One of the main reasons for the dc resonances to appear at different frequencies for the MMC-based scheme compared to the two-level case, is the difference in dc system characteristics (mainly the converter's $c_{d c}$ ). Furthermore, more interactions are seen for the case of the MMCs due to possible interactions with the internal converter dynamics.

Fig. 9 shows the results of an MMC-based scheme with the pole-to-ground dc capacitance $C_{d c, p t g}$ at each terminal equal to the value for the two-level converter. A first observation is that the resonance frequencies of the dc modes are largely corresponding to those of the two-level scheme. Furthermore, the number of interactions passing the threshold somewhat reduces. Indeed, modes $A, B$ (respectively $\mathrm{A}^{\prime}, \mathrm{B}^{\prime}$ ) are located at the same position (Fig. 9) and show a similar component participation (Figs. 8a and 10). A similar remark holds for the modes $\mathrm{C}^{\prime}, \mathrm{D}^{\prime}$ in the two-level scheme and modes $G, H$. Modes $C$ to $F$ are linked with the circulating current $i_{c, z}$ and the energy-sum $w_{\Sigma}$ dynamics and correspond to the poles $G$ to $J$ in Fig. 7. Adding the capacitances at both cable end also has an effect on the modes associated with the voltage control, namely $I, J$ in Fig. 9 and $K, L$ in Fig. 7.

\section{CONCLUSION}

Comparison of the small-signal dynamics in a point-to-point VSC HVDC link based on two-level VSCs and MMCs shows that for the MMC-based topology, more interactions can be present as far as the system behavior is concerned. MMCbased transmission schemes can result in more complex interaction patterns, possibly involving energy-sum and circulating current dynamics in case of active control of the energybalance. This especially holds for converters with similar parameters and control settings. With proper tuning, these oscillating modes are however well-damped and therefore should not affect the overall system behavior in a detrimental way. Similarly, they should not significantly influence the small-signal dynamics of the HVDC transmission scheme as seen from the ac-side networks.

\section{REFERENCES}

[1] N. Kroutikova, C. Hernandez-Aramburo, and T. Green, "State-space model of grid-connected inverters under current control mode," IET Electric Power Applications, vol. 1, no. 3, pp. 329-338, May 2007.

[2] A. Yazdani and R. Iravani, "A generalized state-space averaged model of the three-level NPC converter for systematic DC-voltage-balancer and current-controller design," IEEE Trans. Power Del., vol. 20, no. 2, pp. 1105-1114, Apr. 2005.

[3] G. O. Kalcon, G. P. Adam, O. Anaya-Lara, S. Lo, and K. Uhlen, "Smallsignal stability analysis of multi-terminal VSC-based DC transmission systems," IEEE Trans. Power Syst., vol. 27, no. 4, pp. 1818-1830, Nov. 2012.

[4] N. Chaudhuri, R. Majumder, B. Chaudhuri, and J. Pan, "Stability analysis of VSC MTDC grids connected to multimachine AC systems," IEEE Trans. Power Del., vol. 26, no. 4, pp. 2774-2784, Oct. 2011.

[5] A. Lesnicar and R. Marquardt, "An innovative modular multilevel converter topology suitable for a wide power range," in Proc. IEEE PowerTech 2003, vol. 3, Bologna, Italy, Jun. 23-26, 2003, pp. 272-277.

[6] G. Bergna Diaz, J. Suul, and S. D'Arco, "Small-signal state-space modeling of modular multilevel converters for system stability analysis," in Proc. IEEE Energy Conversion Congress and Exposition (ECCE) 2015, Montreal, Canada, Sep. 20-24, 2015, pp. 5822-5829.

[7] A. Far and D. Jovcic, "Small signal dynamic dq model of modular multilevel converter for system studies," IEEE Trans. Power Del., 2015, in press.

[8] J. Beerten, S. D'Arco, and J. A. Suul, "Cable model order reduction for HVDC systems interoperability analysis," in Proc. IET ACDC 2015, Birmingham, UK, Feb. 10-12, 2015, 10 pages.

[9] J. Beerten, S. D'Arco, and J. Suul, "Identification and small-signal analysis of interaction modes in VSC MTDC systems," IEEE Trans. Power Del., 2015, in press.

[10] S. D'Arco, J. A. Suul, and M. Molinas, "Implementation and analysis of a control scheme for damping of oscillations in VSC-based HVDC grids," in Proc. PEMC 2014, Antalya, Turkey, Sep.,21-24, 2014, 8 pages.

[11] J. Beerten, S. D'Arco, and J. A. Suul, "Frequency-dependent cable modelling for small-signal stability analysis of VSC HVDC systems," IET Generation, Transmission and Distribution, in press.

[12] W. Leterme, N. Ahmed, J. Beerten, L. Ängquist, D. Van Hertem, and S. Norrga, "A new HVDC grid test system for HVDC grid dynamics and protection studies in EMT-type software," in Proc. IET ACDC 2015, Birmingham, UK, Feb. 10-12, 2015, 7 pages.

[13] J. Peralta, H. Saad, S. Dennetière, J. Mahseredjian, and S. Nguefeu, "Detailed and averaged models for a 402-level MMC-HVDC system," IEEE Trans. Power Del., vol. 27, no. 3, pp. 1501-1508, Jul. 2012.

[14] P. Kundur, Power System Stability and Control. McGraw-Hill Inc, New York, 1993. 